\title{
Evaluation of the interactions between human serum albumin (HSA) and warfarin or diflunisal by using molecular fluorescence using two approaches
}

\author{
Susana Amézqueta, Anna Maria Bolioli, José Luis Beltrán, Clara Ràfols* \\ Departament d'Enginyeria Química i Química Analítica and Institute of Biomedicine of the University of Barcelona \\ (IBUB), Universitat de Barcelona, Martí i Franquès 1-11, 08028, Barcelona, Spain
}

*Corresponding Author: E-mail: crafols@ub.edu; Tel.: +34-934-034-874; Fax: +34-934-021-233

Received: November 03, 2017; Revised: January 12, 2018; Published: March 25, 2018

\begin{abstract}
Serum albumin is the main drug transporter of the bloodstream and contains two main binding sites: Sudlow I or acidic drug binding site, and Sudlow II or benzodiazepine binding site. Warfarin, a well-known anticoagulant drug commonly used in the prevention of thrombosis and thromboembolism, binds to Sudlow I site, whereas non-steroidal antiinflammatory drugs (NSAIDs) such as diflunisal bind preferentially to Sudlow II site. Albumin is a fluorophore that modifies its fluorescence (quenching or enhancement effect) when it is bound to a drug. The application of the double logarithm Stern-Volmer equation allows the calculation of the stoichiometry and the binding constant of the process. This procedure does not consider the possible interferences coming from the fluorescence of the drug though. Another strategy to evaluate the binding constants is to consider the whole spectrum, taking into account all the possible species in equilibrium; in this case we have used an extended version of the STAR program, which can deal with 300 spectra, each containing up to 300 data points. The aim of this work is to compare both approaches to evaluate the interaction between warfarin (Sudlow I) and diflunisal (Sudlow II) and HSA: the double logarithm Stern-Volmer equation and the STAR program.
\end{abstract}

\section{Keywords}

HAS; protein-albumin interaction; fluorescence; warfarin; NSAIDs

\section{Introduction}

Albumin, the most abundant protein in plasma and serum, is a water-soluble macromolecule with high biological significance. It is able to maintain the plasma oncotic pressure and modulate the fluid distribution among body compartments. Native albumin, that is the one without ligands or bound molecules, is built up from three homologous domains (I, II and III), showing each one two distinct subdomains, named $A$ and $B$. There are numerous binding sites on albumin, but drugs and other exogenous compounds bind, mainly, to two of them: Sudlow I or acidic drug binding site, placed on subdomain IIA, and Sudlow II or benzodiazepine binding site, located on subdomain IIIA. Thus, albumin plays a relevant role in binding and transport functions and, therefore, in the pharmacokinetics of drugs [1].

Drug-albumin interactions can be evaluated using several complementary techniques such as 
isothermal titration calorimetry, fluorescence, frontal analysis capillary electrophoresis or equilibrium dialysis $[2,3]$. In the case of fluorescence, the albumin is considered as the main fluorophore, as it contains three fluorescent amino acids (tryptophan, tyrosine and phenylalanine). When the complex drug-protein is formed, the albumin fluorescence can be either quenched or enhanced. There are some equations to evaluate the fluorescence quenching/enhancement that allow calculating the binding parameters (binding constant and stoichiometry) [4]. These equations present several drawbacks, as they consider the albumin as the unique fluorophore, and assume that only one type of interaction is formed and that the concentration of the free drug is much higher than the bound fraction. However, there often exist other fluorophores in solution (such as the drug or a drug-albumin complex) that force to work under more selective but less sensitive conditions. Another strategy to evaluate the binding constants is to consider the whole fluorescence spectrum, taking into account all the possible species in equilibrium.

The aim of this work is to evaluate the interaction between warfarin, interacting into Sudlow I, or diflunisal, interacting into Sudlow II with human serum albumin (HSA) by fluorescence, using both approaches to calculate the binding parameters and compare the results obtained.

\section{Experimental}

HSA (99\%), warfarin (>99\%), and diflunisal (>98 \%) were obtained from Sigma-Aldrich (St Louis, MO, United States). Hydrochloric acid (0.5 M), sodium dihydrogen phosphate monohydrate (>99\%), disodium hydrogen phosphate ( $>99 \%$ ), sodium chloride (>99\%), and potassium chloride (>99\%) were from Merck (Darmstadt, Germany). Water was purified by a Milli-Q plus system from Millipore (Bedford, MA, United States), with a resistivity of $18.2 \mathrm{M} \Omega \mathrm{cm}$. PBS (phosphate buffer solution) (10 mM, I=150 mM) consisted of an aqueous solution containing $0.137 \mathrm{M} \mathrm{NaCl}, 0.0027 \mathrm{M} \mathrm{KCl}, 0.0015 \mathrm{mM} \mathrm{KH_{2 }} \mathrm{PO}_{4} \cdot 3 \mathrm{H}_{2} \mathrm{O}$ and $0.0081 \mathrm{M}$ $\mathrm{Na}_{2} \mathrm{HPO}_{4} \cdot \mathrm{H}_{2} \mathrm{O}$. The solution $\mathrm{pH}$ was adjusted to 7.40 with $0.5 \mathrm{M} \mathrm{HCl}$. Drugs and albumin were dissolved in PBS at the desired concentration (300 - $1000 \mu \mathrm{M}$ and $3 \mu \mathrm{M}$, respectively).

Quenching studies were carried out by titration over a HSA solution $(2.7 \mathrm{~mL})$ with an initial concentration of $3 \mu \mathrm{M}$. The albumin was placed in a fluorescence cuvette at $25^{\circ} \mathrm{C}$. The initial fluorescence spectrum was recorded. Sixteen successive additions of the drug under study (warfarin, or diflunisal) were done to cover a drug concentration in the range $0.4-14 \mu \mathrm{M}$. After each addition, the solution in the cuvette was stirred to allow the temperature and reaction equilibration. Then, the fluorescence spectrum was acquired using a Cary Eclipse fluorimeter from Agilent Technologies (Santa Clara, MA, United States) [4] using $5 \mathrm{~nm}$ slits in both, excitation and emission monochromators. These protein and drug concentrations allow measuring the specific binding of the drug to the protein and do not correspond to the plasmatic ones. In fact, the usual albumin concentration in plasma is about $600 \mu \mathrm{M}$ and the drug:albumin ratio is usually as low as 1:150 for warfarin (30 mg/week) [5] and 1:2 for diflunisal (500 mg, single dose) [6].

\section{Data treatment}

Fluorescence data were recorded in three ways: a standard fluorescence spectrum, and as synchronous fluorescence spectra at $\Delta \lambda=15 \mathrm{~nm}$ and $\Delta \lambda=60 \mathrm{~nm}$ for the selective measurement of the fluorophores tyrosine and tryptophan, respectively.

To evaluate the stoichiometry and the binding constant, the double logarithm Stern-Volmer equation (DLSV) was considered in first instance (Eq. 1) [4]. 


$$
\log \frac{F_{0}-F}{F}=\log K_{\mathrm{b}}+n_{H} \log \left[Q_{\mathrm{f}}\right]
$$

where $F_{0}$ and $F$ are the fluorescence intensity in the absence and the presence of a quencher, respectively; $K_{\mathrm{b}}$ is the binding constant; $n_{\mathrm{H}}$ is the Hill coefficient; and $\left[Q_{\mathrm{f}}\right]$ is the free quencher (drug) concentration. Usually, the free drug concentration $\left[Q_{\mathrm{f}}\right]$ is unknown and it is replaced by the total drug concentration $[Q]$ (it is assumed that the concentration of the macromolecule is negligible with respect to the drug one) (Eq. 2). Moreover, this equation only yields the binding stoichiometry in case of "infinite" cooperativity [7].

$$
\log \frac{F_{0}-F}{F}=\log K_{\mathrm{b}}+n_{H} \log [Q]
$$

The substitution of $\left[Q_{f}\right]$ by $[Q]$ indicates that the DLSV equation should be applied only when the solution contains a large excess of drug. On the other hand, it is also assumed that the fluorescence intensity of the drug is negligible. Therefore, the applicability of the DLSV equation is restricted to these scenarios. In order to overcome both problems we have applied an extended version of the non-linear least squares program STAR [8], which can handle the spectroscopic data from multiple-equilibria systems containing up to 300 solutions, measured at 300 data points. In this case, an equilibrium system can be described by a set of components (as albumin, quencher, hydrogen ion, etc.) which can form different species. These species are defined by their stoichiometric coefficients and the corresponding binding constants:

$$
\mathrm{sS}+\mathrm{qQ} \rightleftarrows \mathrm{S}_{\mathrm{s}} \mathrm{Q}_{\mathrm{q}} \quad K_{\mathrm{b}}=\frac{\left[S_{s} Q_{q}\right]}{[S]^{\mathrm{s}}[Q]^{\mathrm{q}}} .
$$

STAR uses a chemical equilibrium model, which consists of the components of the system (e.g., HSA and drug), together with the guessed stoichiometry and binding constants of the species formed. Moreover, the experimental data for each solution contains the total concentrations of components and the measured fluorescence spectrum.

On the other hand, we can consider that the fluorescence spectrum of a given solution is equal to the sum of the fluorescence spectra of all species. If we have a set of $n$ fluorescent species, STAR solves the mass balances of the components calculating the concentrations for all species for the given set of binding constants. Then, the fluorescence intensity at a $j$ wavelength can be calculated as:

$$
I f_{\mathrm{j}}=\sum_{i=1}^{n} c_{\mathrm{i}} \phi_{\mathrm{i}, \mathrm{j}},
$$

where $C_{i}$ indicates the concentration of the $i$-species, and $\phi_{i, j}$ the relative fluorescence of the $i$-species at the $j$-wavelength. The $\phi_{1, j}$ values can be supplied as data input, or calculated by the program.

The binding constants are refined by the Gauss-Newton iterative algorithm, until a minimum of the sum of squared differences $(U)$ between the experimental and calculated fluorescence intensities is obtained:

$$
U=\sum_{i=1}^{n s} \sum_{i=1}^{n w}\left(I f_{i, j, \exp }-I f_{i, j, c a l c}\right)^{2}
$$

where $n s$ and $n w$ indicate the number of solutions and wavelengths, respectively; the subindexes exp and calc indicate experimental values and calculated by the program.

The program output includes the standard deviation of the errors between experimental and calculated 
data, together with the estimated error in the binding constants.

STAR allows the data treatment of the full fluorescence spectra (as it can handle up to 300 solutions, measured up to 300 data points in each spectrum), calculating the binding constants and the unitary spectrum of the species formed. In this way, the drawbacks owed to the application of the DLSV equation are easily overcome.

\section{Results and Discussion}

When a successive amount of the studied drugs is added to a fixed concentration of HSA, the albumin fluorescence is quenched; this is the case of the HSA fluorescence band at about $285 \mathrm{~nm}$. As an example, Figure 1 shows the changes in the synchronous fluorescence spectra $(\Delta \lambda 60 \mathrm{~nm})$ for HSA after the addition of diflunisal.

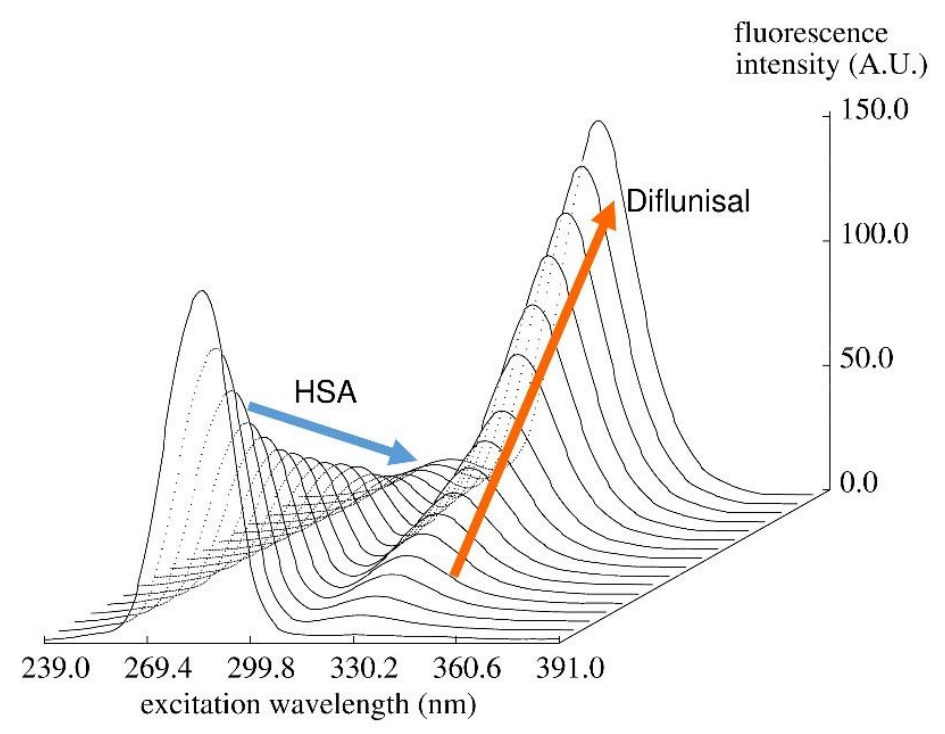

Figure 1. Quenching study of diflunisal-HSA at synchronous $\Delta \lambda 60 \mathrm{~nm}$

The DLSV approach uses the fluorescence intensity value of the fluorophore at a fixed wavelength, usually the one that offers the maximum intensity. As the drugs used in the study may be fluorescent in the working conditions, an initial study of interferences has been performed in the three modes selected. HSA shows maximum fluorescence in the emission mode at 340-350 nm, and in the synchronous modes at 285$288 \mathrm{~nm}$. At these regions, diflunisal does not interfere with HSA, while warfarin (Figure 2) interferes about $9 \%$ in the emission mode, over $10 \%$ in synchronous $\Delta \lambda 60 \mathrm{~nm}$ mode and does not interfere in synchronous $\Delta \lambda 15 \mathrm{~nm}$ mode. Although in some cases interferences exist, we have selected the wavelength with maximum fluorescence intensity for each drug and mode to evaluate the results. We assume that the interferences under $10 \%$ can be evaluated using the DLSV equation. The working conditions are indicated in Table 1.

First, the fluorescence data have been evaluated with the DLSV equation considering the range of linear response (0.4-5 or 0.4-14 $\mu \mathrm{M}$, depending on the drug and the fluorescence mode). The results obtained show that diflunisal and warfarin interact with HSA with a log $K_{\mathrm{b}}$ value about 5 (Table 2). For diflunisal, not significant differences in the log $K_{\mathrm{b}}$ values have been observed when evaluating the data at the three modes of acquisition. In the case of warfarin, the log $K_{b}$ calculated considering the emission mode (where warfarin fluorescence interferes about $9 \%$ ) is slightly lower than the one obtained taking into account the synchronous $\Delta=15 \mathrm{~nm}$ mode (without interferences). 

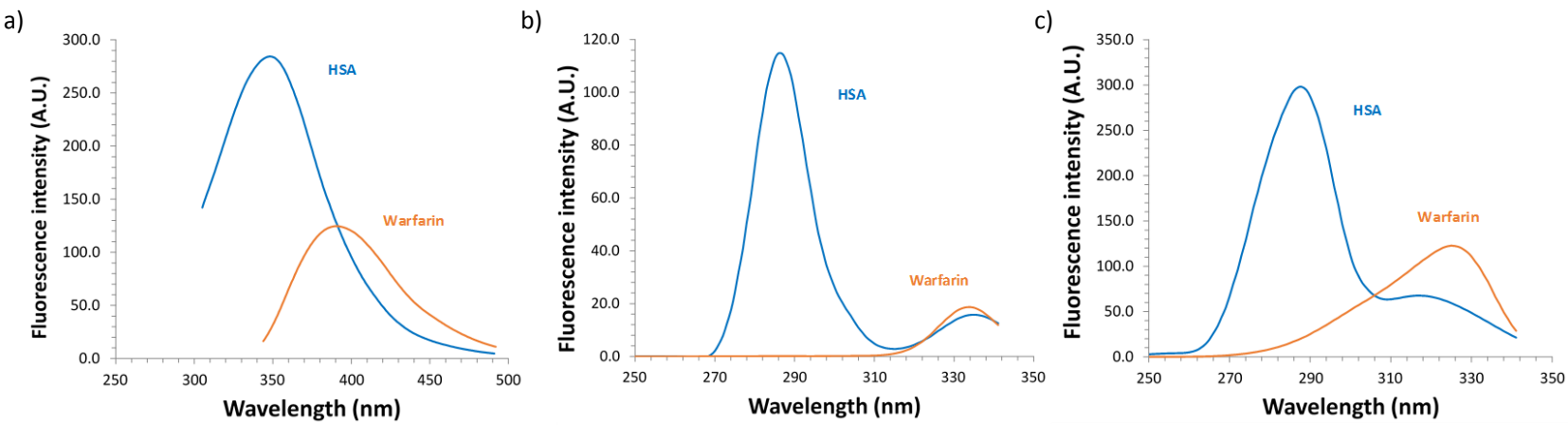

Figure 2. Comparison of fluorescence spectra in the study of warfarin-HSA: (a) emission, (b) synchronous $\Delta \lambda 15 \mathrm{~nm}$ and (c) synchronous $\Delta \lambda 60 \mathrm{~nm}$ modes

Table 1. Optimum wavelengths found for the evaluation of the drug-HSA interaction for the three modes of interest.

\begin{tabular}{lcc} 
& Warfarin & Diflunisal \\
\hline Emission, $\lambda$ ex $=285 \mathrm{~nm}$ & $350 \mathrm{~nm}$ & $348 \mathrm{~nm}$ \\
Synchronous $\Delta \lambda 15 \mathrm{~nm}$ & $285 \mathrm{~nm}$ & $286 \mathrm{~nm}$ \\
Synchronous $\Delta \lambda 60 \mathrm{~nm}$ & - & $288 \mathrm{~nm}$ \\
\hline
\end{tabular}

The binding constants have been also evaluated with the modified STAR program. The results are also shown in Table 2. In the case of diflunisal, which does not present interferences at the working conditions, the results obtained with this program (restricting the model to a 1:1 binding equilibrium) are similar to the ones obtained by DLSV equation and are similar at the three modes evaluated. For warfarin, the results calculated with the STAR program agree with those obtained with the DLSV approach when there are not interferences (synchronous $\Delta \lambda 15 \mathrm{~nm}$ ). When interferences exist (emission mode), the STAR program is able to deal with them and offers $\log K_{b}$ values similar to those obtained in the absence of interferences.

Moreover, the use of STAR program has allowed detecting the presence of two interactions between HSA and diflunisal when it is assumed the possible presence of more than one kind of interaction (Table 2, last row). In fact, literature reports that diflunisal can bind to two different sites with different affinity [9]. For this drug, the first log $\mathrm{K}_{\mathrm{b}}$ value is slightly higher than the one obtained by DLSV equation, but when the equilibrium model in STAR contains only one interaction, the results agree with those obtained by DLSV equation. In this case, the calculated value by STAR for $\log \mathrm{K}_{\mathrm{b}}$ is intermediate between $\log \mathrm{K}_{\mathrm{b} 1}$ and $\log \mathrm{K}_{\mathrm{b} 2}$, in order to accomplish the mass balances of the components. Therefore, when multiple species are involved in the binding process, the DLSV approach allows calculating the predominant event, whereas the STAR program allows the study of all species in equilibrium. 


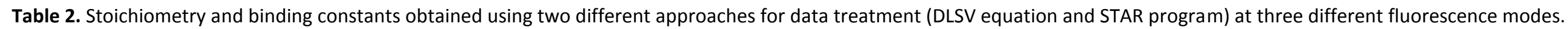

\begin{tabular}{|c|c|c|c|c|c|c|c|c|c|c|c|c|c|}
\hline & & \multicolumn{4}{|c|}{ Emission } & \multicolumn{4}{|c|}{ Synchronous $\Delta \lambda 15 \mathrm{~nm}$} & \multicolumn{4}{|c|}{ Synchronous $\Delta \lambda 60 \mathrm{~nm}$} \\
\hline & & $\mathrm{n}_{1}$ & $\log K_{\mathrm{b} 1}$ & $\mathrm{n}_{2}$ & $\log K_{\mathrm{b} 2}$ & $\mathrm{n}_{1}$ & $\log K_{\mathrm{b} 1}$ & $\mathrm{n}_{2}$ & $\log K_{b 2}$ & $\mathrm{n}_{1}$ & $\log K_{\mathrm{b} 1}$ & $\mathbf{n}_{2}$ & $\log K_{\mathrm{b} 2}$ \\
\hline \multirow{2}{*}{$\begin{array}{c}\text { HSA- } \\
\text { Warfarin }\end{array}$} & DLSV & $0.98(0.02)$ & $4.6(0.2)^{a}$ & & & $1.05(0.07)$ & $5.0(0.3)^{a}$ & & & - & - & & \\
\hline & STAR & 1 & $5.14(0.01)$ & & & 1 & $4.97(0.02)$ & & & - & - & & \\
\hline \multirow{3}{*}{$\begin{array}{c}\text { HSA- } \\
\text { Diflunisal }\end{array}$} & DLSV & $1.01(0.02)$ & $5.2(0.1)^{a}$ & & & $1.09(0.02)$ & $5.1(0.1)^{a}$ & & & $1.04(0.02)$ & $5.3(0.1)^{\mathrm{a}}$ & & \\
\hline & $S T A R^{b}$ & 1 & $5.18(0.01)$ & & & 1 & $4.90(0.02)$ & & & 1 & $5.34(0.01)$ & & \\
\hline & STAR & 1 & $5.8(0.2)$ & 2 & $4.7(0.1)$ & 1 & $5.44(0.04)$ & 2 & $4.6(0.1)$ & 1 & $5.7(0.1)$ & 2 & $4.5(0.2)$ \\
\hline
\end{tabular}

${ }^{a}$ : determination coefficient $\left(R^{2}\right) \sim 0.98 ;^{b}$ : the model is restricted to $1: 1$ binding equilibria

Table 3. Stoichiometry and binding constants reported in the literature for warfarin or diflunisal interactions with HSA.

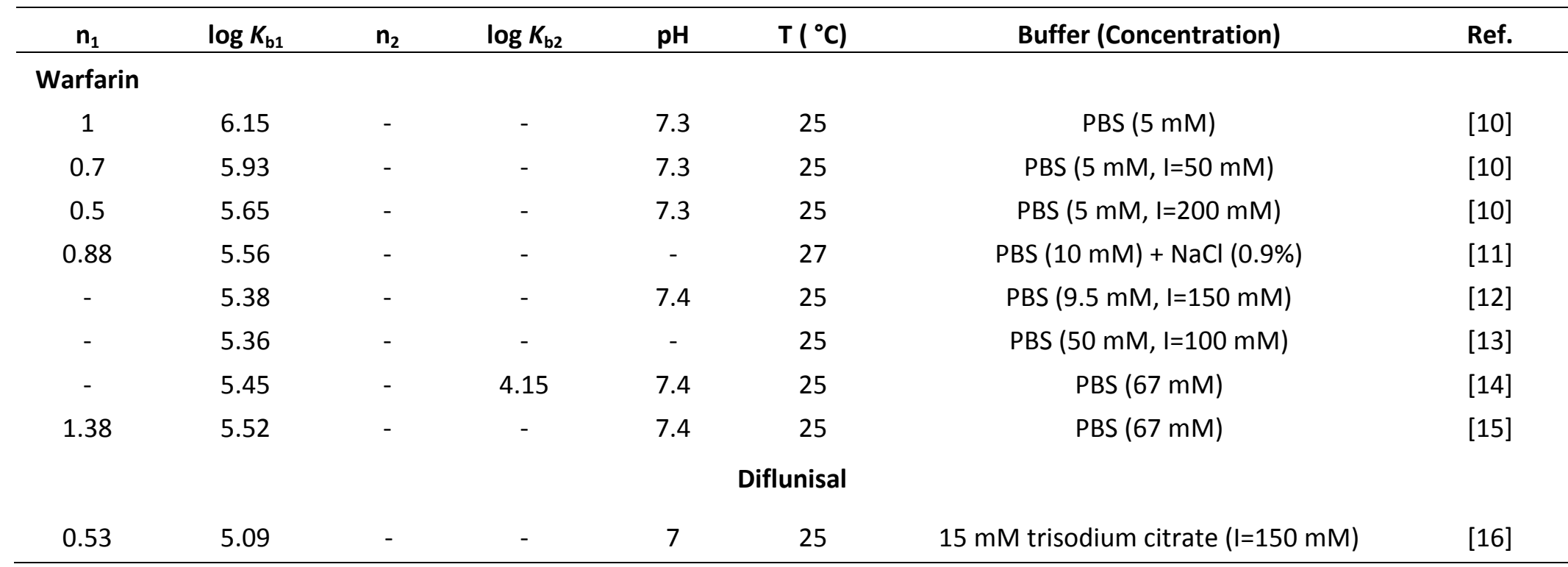


Compared to the results reported by other authors (Table 3) using fluorescence and different experimental conditions, the stoichiometry agrees with the reported data ( $\mathrm{n}$ in the range 0.5-1.38 $\mu \mathrm{M}$ ). The log $\mathrm{K}_{\mathrm{b}}$ values obtained in the present work are slightly lower than those obtained by other authors. Note that higher ionic strength implies lower log $K_{b}$ values and therefore the experimental conditions used can be meaningful. In the case of diflunisal, the results reported by Davilas et al. [17] agree with the ones obtained in the present work when a unique species is considered. Another work on diflunisal interaction with HSA [17], using the internal standard electrode method, reports two interactions $\left(\log K_{\mathrm{b} 1}=5.7\right.$, $\left.\log K_{\mathrm{b} 2}=3.8\right)$ and agrees with the results obtained with the STAR program considering the presence of several species.

\section{Conclusions}

HSA interaction with warfarin and diflunisal has been evaluated using fluorescence measurements. First, an interference study has been carried out showing that warfarin interferes under the working conditions, while diflunisal does not. Next, the drug-albumin interaction has been studied using two different approaches. These drugs bind to the albumin with $a K_{b}$ in the order of $10^{5}$. In the absence of interferences or when a unique species is considered (stoichiometry 1:1), both approaches yield similar values. The modified STAR program is also able to deal with the interferences of fluorescent drugs and can consider multiple species. In the case of warfarin, the log $K_{b}$ values calculated agree in the absence and presence of interferences, and in the case of diflunisal it shows that there are two different processes involved, with stoichiometry 1:1 and 1:2.

Acknowledgements: We thank Spanish Government for the research grant (project CTQ2014-56253-P).

\section{References}

[1] K. Yamasaki, V.T.G. Chuang, T. Maruyama, M. Otagiri. Albumin-drug interaction and its clinical implication. Biochimica et Biophysica Acta 1830 (2013) 5435-5443.

[2] S.E. Harding, B.Z. Chowdhry, Protein-ligand interactions: hydrodynamics and calorimetry, Oxford University Press: New York 2011.

[3] C. Ràfols, S. Zarza, E. Bosch. Molecular interactions between some non-steroidal anti-inflammatory drugs (NSAID' s) and bovine (BSA) or human (HSA) serum albumin estimated by means of isothermal titration calorimetry (ITC) and frontal analysis capillary electrophoresis (FA/CE). Talanta 130 (2014) 241-250.

[4] J. Yang, L. Qu, W. Y, Y. Huang, N. Jiao, W. Zhan, D. Zhao, L. Cui. Interaction of hyperoside with human serum albumin and effect of glucose on the binding. Journal of Spectroscopy 386586 (2014) 1-9.

[5] T. Lomonaco, S. Ghimenti, I. Piga, D. Biagini, M. Onor, R. Fuoco, A. Paolicchi, L. Ruocco, G. Pellegrini, M.G. Trivella, F. Di Francesco. Monitoring of warfarin therapy: Preliminary results from a longitudinal pilot study. Microchem. J. 136 (2017) 170-176.

[6] D.S. Patel, N. Sharma, M.C. Patel, B.N. Patel, P.S. Shrivastav, M. Sanyal. Sensitive and selective determination of diflunisal in human plasma by LC - MS. J. Chromatogr. Sci. 51 (2013) 872-882.

[7] G. Li, B.S. Liu, Q. Zhang, R. Han. Investigation on the effect of fluorescence quenching of bovine serum albumin by cefoxitin sodium using fluorescence spectroscopy and synchronous fluorescence spectroscopy. Luminiscence 31 (2016) 1054-1062.

[8] J.L. Beltrán, R. Codony, M.D. Prat. Evaluation of stability constants from multi-wavelength absorbance data: program STAR. Analytica Chimica Acta 276 (1993) 441-454.

[9] J. Ghuman, P.A. Zunszain, I. Petitpas, A.A. Bhattacharya, M. Otagiri, S. Curry. Structural basis of the drug-binding specificity of human serum albumin. Journal of Molecular Biology 353 (2005) 38-52. 
[10] P. Bolel, S. Datta, N. Mahapatra, M. Halder. Exploration of $\mathrm{pH}$-dependent behavior of the anion receptor pocket of subdomain IIA of HSA: determination of effective pocket charge using the DebyeHückel limiting law. Journal of Physical Chemistry B 118 (2014) 26-36.

[11] V. Maes, Y. Engelborghs, J. Hoebeke, Y. Maras, A. Vercruysse. Fluorimetric analysis of the binding of warfarin to human serum albumin. Equilibrium and kinetic study. Molecular Pharmacology 21 (1982) 100-107.

[12] M. Poór, Y. Li, S. Kunsági-Máté, J. Petrik, S. Vladimir-Knežević, T. Kőszegi, T. Koszegi. Molecular displacement of warfarin from human serum albumin by flavonoid aglycones. Journal of Luminiscence 142 (2013) 122-127.

[13] C. Dufour, O. Dangles. Flavonoid-serum albumin complexation: determination of binding constants and binding sites by fluorescence spectroscopy. Biochimica Biophysica Acta - Gen. Subj. 1721 (2005) 164-173.

[14] M. Dockal, M. Chang, D.C. Carter, F. Rüker. Five recombinant fragments of human serum albumintools for the characterization of the warfarin binding site. Protein Science 9 (2000) 1455-1465.

[15] R. Abou-Khalil, A. Jraij, J. Magdalou, N. Ouaini, D. Tome, H. Greige-Gerges. Interaction of cucurbitacins with human serum albumin: Thermodynamic characteristics and influence on the binding of site specific ligands. Journal of Photochemistry Photobiology B 95 (2009) 189-195.

[16] S. Fountoulaki, F. Perdih, I. Turel, D.P. Kessissoglou, G. Psomas. Non-steroidal anti-inflammatory drug diflunisal interacting with $\mathrm{Cu}(\mathrm{II})$. Structure and biological features. Journal of Inorganic Biochemistry 105 (2011) 1645-1655.

[17] A. Davilas, M. Koupparis, P. Macheras, G. Valsami. In-vitro study on the competitive binding of diflunisal and uraemic toxins to serum albumin and human plasma using a potentiometric ion-probe technique. Journal of Pharmacy and Pharmacology 58 (2006) 1467-1474. 